\title{
Judgment of Psychological Fatigue and Physiological Fatigue based on HRV
}

\author{
Duan Jing Le \\ Tianjin University of Technology and Education \\ Institute of robotics and intelligent equipment \\ Tianjin , P. R.China \\ Sun Guifen \\ Tianjin University of Technology and Education \\ Tianjin , P. R.China
}

\author{
Qi Yu Ming \\ Tianjin University of Technology and Education \\ Institute of robotics and intelligent equipment \\ Tianjin , P. R.China \\ Yue Gang \\ Tianjin Transportation Technical College \\ Tianjin , P. R.China
}

\begin{abstract}
According to the health survey of middle-aged people, every year millions of people die from excessive fatigue, and $60 \%$ of them suffer from chronic fatigue syndrome such as insomnia, backache and memory decline. It can be seen that fatigue poses a great threat to people's physical and mental health. This paper intends to conduct physiological fatigue and psychological fatigue experiments, monitor ECG signals, extract HRV, get LF, and judge the difference between the two fatigue. This method is non-invasive and operable, and provides an effective functional tool for fatigue monitoring.
\end{abstract}

Keywords-VDT Visual Fatigue; Cardiac Impulse Signal Extraction; Unconstrained Monitoring; Adaptive Threshold Wavelet Denoising

\section{INTRODUCTION}

According to the health survey of middle-aged people, every year millions of people die from excessive fatigue, and $60 \%$ of them suffer from chronic fatigue syndrome such as insomnia, backache and memory decline. It can be seen that fatigue poses a great threat to people's physical and mental health. However, fatigue can be divided into three types according to the cause of disease: physical fatigue, physiological fatigue and psychological fatigue. Among them, physical fatigue can reduce the workload and recover in a short time; however, physical fatigue and psychological fatigue have a great impact on people, and the treatment cycle is long and difficult. Therefore, this paper mainly studies physiological and psychological fatigue. At present, there are three main research methods of physical fatigue and mental fatigue: observation evaluation, subjective sensory evaluation and objective evaluation. Among them, the observation evaluation

\section{EXPERIMENTAL SETTINGS}

In this chapter, physiological fatigue and psychological fatigue induction experiments are carried out, ECG signals are monitored, HRV is extracted, LF is obtained, and the difference between the two fatigues is judged. This method will not cause trauma to the tester, and provides an effective functional tool for fatigue monitoring. method and subjective sensory evaluation method are mainly based on the judgement of the observer or the self-evaluation of the subjects. The multi-dimensional fatigue scale (MFI$20)^{[1]}$ is used to evaluate the fatigue of diabetic patients in three dimensions: physical fatigue, psychological fatigue and mental fatigue, which has good reliability and validity. However, it is subjective and can only be used as an auxiliary evaluation method. The objective evaluation method is mainly based on the dynamic changes of material metabolism, autonomic nervous system and brain area. Tanaka ${ }^{[2]}$ and Konstandi $\mathrm{M}^{[3]}$ both induce mental fatigue in healthy subjects, and monitor and record the activity of autonomic nervous system using ECG and EEG detection equipment respectively. The activity of vagus nerve decreases and sympathetic nerve increases when the subjects suffer from mental fatigue ${ }^{[4]}$, but it is not clear whether physiological fatigue will lead to this phenomenon. The causes, measurement methods and manifestations of physical fatigue and mental fatigue were studied. Guo Siyuan ${ }^{[5]}$ preliminarily explored the pathogenesis of mental fatigue from four aspects: autonomic nervous system, material metabolism, sensorimotor gating and brain area changes. However, the research status was summarized by consulting data, and no experimental verification was carried out.

It can be seen that if we reduce the harm of fatigue to people's body, we need to study the difference between physiological fatigue and psychological fatigue leading to ECG, and carry out targeted treatment. This paper intends to conduct physiological fatigue and psychological fatigue experiments, monitor ECG signals, extract HRV, get LF, and judge the difference between the two fatigue. This method is non-invasive and operable, and provides an effective functional tool for fatigue monitoring.

In order to further improve the accuracy of the experiment, SD subjective evaluation was carried out simultaneously in the process of inducing two kinds of fatigue. 


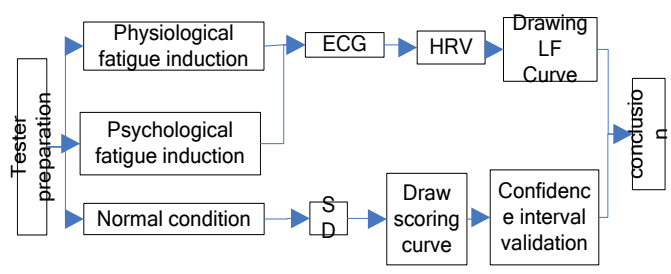

Fig. 1 Experimental design flow chart

\section{A. Tester Requirements}

Nine undergraduates, aged 20-25 years, without disease, psychological and physiological fatigue, body weight: 142.62 $(+10.54)$, were selected as subjects. They were required not to take any drugs or food that affected people's mental health three days before the experiment.

\section{B. Maintaining Normal State}

The test time was 19 hours (21:00-21:00 the next day). The tasks of the subjects were as follows: 21:00-22:00: washing; 22:00-7:00: sleep. 7:00-7:30 to get up and wash; 7:30-8:00 to exercise properly (no strenuous exercise); 8:00-8:30 to eat; 8:30-12:00 to read the paper book Gone with the Wind, take a 10-minute rest every hour; $12: 00-12: 30$ to eat; $12: 30-13: 00$ to take a proper rest; 13:00-13:30 to take a lunch break; 13:3016:00 to take a rest;

Watch the TV series "Railway Guerrillas" and rest for 15 minutes every hour. ECG monitoring is carried out while doing tasks.

\section{Physiological Fatigue Induction}

Sleep deprivation combined with physical exercise was used to induce physiological fatigue. The test time was 15 hours (21:00-12:00 the next day). The task of the tester: 21:007:00: Staying up late to watch TV series "Railway Guerrilla" or listen to music (to reduce the emotional or subjective incompatibility of the tester, reduce the possibility of physical fatigue being disturbed), wake up in time when the tester is sleepy, and make the total sleep time less than 4 hours. After 9:00 the next day, we will carry out $3-5 \mathrm{~km}$ jogging, badminton, football and other sports activities. The total time of sports is not less than 3 hours. ECG monitoring is carried out while doing tasks.

\section{Induction of Mental Fatigue}

A 90-minute UK task (abbreviated as Uchida-Kraepelin) was used to calculate the mental fatigue induced by the test.
The participants were familiar with the UK workflow 2 days in advance and practiced. They had good sleep quality (sleep duration ( $>8$ hours) three days before the experiment. UK was performed at 9:00-10:30 and ECG was monitored synchronously.

\section{E. Laboratory Equipment and Installation}

PC-80B high-speed ECG detector (as shown in Fig. 2), sampling frequency $512 \mathrm{~Hz}$, equipped with three electrodes, connection mode as shown in Fig. 3.

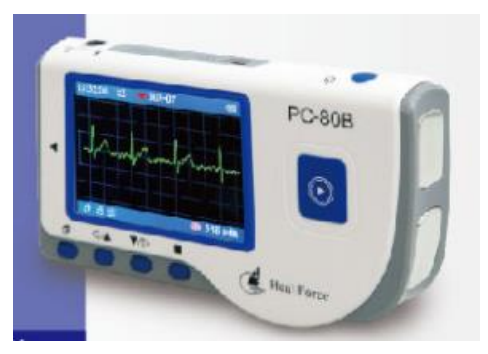

Fig. 2 PC-80B High Speed ECG Detector

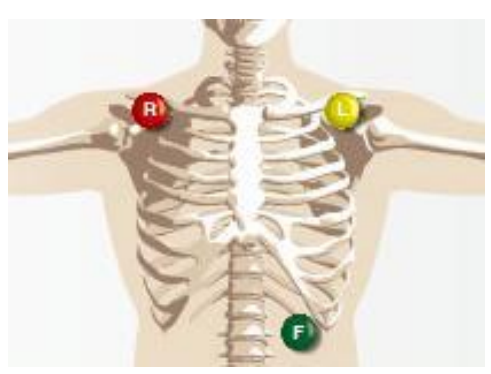

Fig. 3 Electrode connection mode

\section{F. Experimental steps}

All the 10 participants participated in the test. On the first day, they were monitored under normal conditions. On the third day, they were induced by physiological fatigue, and on the third day, they were induced by psychological fatigue.

(1) ECG signal acquisition: ECG signal monitoring in the above three states;

(2) SD subjective sensory assessment (not in normal state): subjective sensory assessment is carried out in the synchronization of ECG signal acquisition.

Among them, according to the Multidimensional Fatigue Inventory (MFI-20) and Burnout Inventory (BM), the SD subjective fatigue assessment table (as shown in Table 1) was modified. There were eight problems. The higher the score, the more serious the fatigue symptoms were. 
TABLE 1 SD SUBJECTIVE FATIGUE ASSESSMENT TABLE

\begin{tabular}{|c|c|c|c|c|c|c|}
\hline & Number: & Time: & & & & \\
\hline & Please mark 0 in $\mathrm{m}$ & tal fatigue or phys & tigue items & & & \\
\hline Psychological fatigue items ( & Physiological fatigue items ( & $\begin{array}{c}\text { Non-conformity } \\
(0 \%)\end{array}$ & $\begin{array}{c}\text { Conform } \\
(25 \%)\end{array}$ & $\begin{array}{c}\text { Middle } \\
(50 \%)\end{array}$ & $\begin{array}{c}\text { Conform } \\
(70 \%)\end{array}$ & $\begin{array}{l}\text { Conform } \\
(100 \%)\end{array}$ \\
\hline 1. Good spirits & 1. Physical comfort & 5 & 4 & 3 & 2 & 1 \\
\hline 2. Energetic & 2. Physical Strength & 5 & 4 & 3 & 2 & 1 \\
\hline 3. Feeling tired & 3.Skeletal discomfort & 1 & 2 & 3 & 4 & 5 \\
\hline 4. Easily distracted. & 4. Muscle soreness & 1 & 2 & 3 & 4 & 5 \\
\hline 5. Physical condition is very good. & 5. Physical condition is very & 5 & 4 & 3 & 2 & 1 \\
\hline 6. Don't want to do anything. & 6. Don't want to move. & 1 & 2 & 3 & 4 & 5 \\
\hline 7. You can do more. & 7. You can continue to & 5 & 4 & 3 & 2 & 1 \\
\hline 8. had a good rest & 8. sleep well & 5 & 4 & 3 & 2 & 1 \\
\hline
\end{tabular}

\section{EXPERIMENTAL DATA PROCESSING}

The ECG data of 10 subjects under normal condition were selected for 5 minutes every 60 minutes for wavelet denoising, HR and LF average calculation. The ECG data under physiological fatigue condition were selected for 5 minutes every 60 minutes for wavelet denoising, HR and LF average calculation, and the SD subjective questionnaire scores were calculated every 60 minutes. Then HR, LF and S were calculated. The D-score curve is drawn in an image, and Fig. 4 and Fig. 4 are obtained. The ECG data under the state of mental fatigue are denoised by wavelet transform every 10 minutes, the average values of HR and LF are calculated, and the scores of SD subjective questionnaire are calculated every 10 minutes. Then the HR, LF and SD score curves are drawn in an image, and Fig. 5 is obtained.

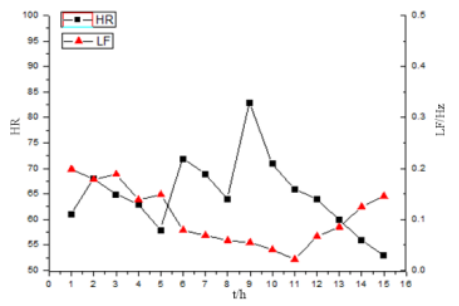

Fig. 4 HR and LF curves under normal conditions

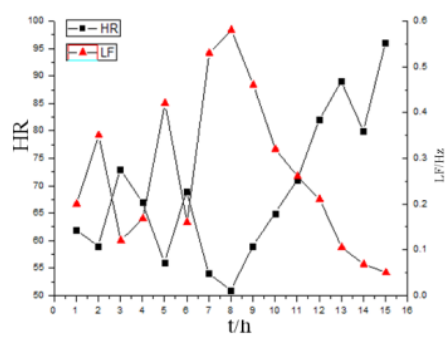

(a) HR and LF curves

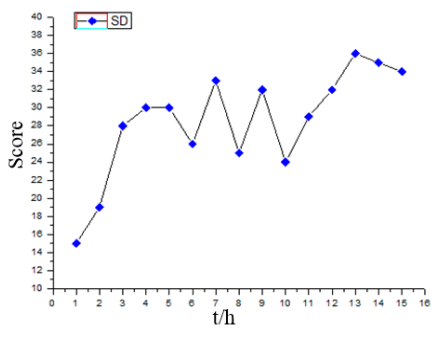

(b) SD curve

Fig. 5 Physiological fatigue curve

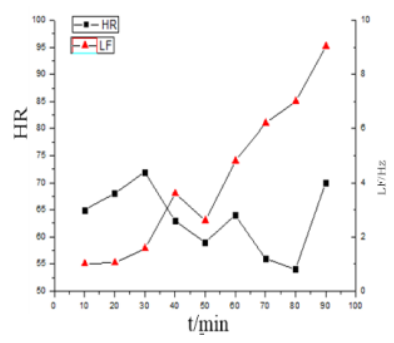

(a) HR and LF Curves

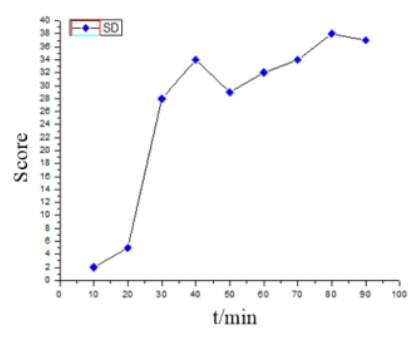

(b) SD curve

Fig. 6 Psychological fatigue curve 
From Fig.6, we can see that HR value increases first and then decreases in the normal state. The main reason is that the tester sleeps from the second hour to the ninth hour. HR value is relatively low and stable. The peak value appears at the tenth hour, which is caused by the physical exercise of the tester, and then reading, which leads to the increase of fatigue and the gradual decrease of HR; LF value decreases first and then increases, from the second hour to the ninth hour. LF is relatively stable after sleeping for hours. The decrease of LF at the 10th hour is due to physical exercise, which leads to a much better mental state of the tester. After reading, fatigue gradually increases and $\mathrm{LF}$ value begins to rise.

From Fig.6, we can see that under physiological fatigue HR value decreases first, then increases, the main trend is to increase, there are some small fluctuations in the middle because the test suddenly wakes up after entering sleep; LF value increases first and then decreases, the main trend is to decrease, some small fluctuations in the middle are because the test suddenly wakes up after entering sleep; SD score increases gradually with the fatigue of the tester. The bigger.

From Fig.6, we can see that the HR value gradually decreases, the LF gradually increases, and the SD score gradually increases with the fatigue of the tester.

\section{ANALYSIS OF EXPERIMENTAL RESULTS}

From the image and the above analysis, we can know that the HR and LF values under physiological fatigue and psychological fatigue are different: with the increase of psychological fatigue, HR decreases and LF increases; with the increase of physiological fatigue, HR increases and LF decreases. The conclusion can be seen that the degree of mental fatigue is negatively correlated with the stress level, while the level of physical fatigue is positively correlated with the stress level, which reflects the self-compensatory adjustment of the body. It can be seen that the activity of autonomic nervous system is different between psychological fatigue and physiological fatigue, which may be related to the difference between the two fatigue and the carrying organs.

\section{CONCLUSION}

This chapter mainly focuses on the difference and judgment between mental fatigue and physiological fatigue, designs a comparative experiment of mental fatigue and physiological fatigue, collects ECG signals, then processes and acquires HRV, and extracts LF. It is found that physical fatigue and psychological fatigue lie in the increase of mental fatigue, the decrease of HR and the increase of HRV; the increase of physiological fatigue, the increase of HR and the decrease of HRV. The research results play an important role in the optimization and upgrading of the discriminant system based on support vector machine in Chapter 4. According to different types of VDT visual fatigue, we can deal with them more pertinently and give reasonable suggestions. For example, if the system judges the samples as mental fatigue, it suggests that users listen to music and do other entertainment activities to eliminate the fatigue; if the system judges the samples as mental fatigue; Physiological fatigue suggests that users take rest or take related sports to eliminate the fatigue.
Of course, the discovery of the difference between psychological fatigue and physiological fatigue will lay a good foundation for future related fatigue research, which can save a lot of time and get research results faster.

\section{ACKNOWLEDGMENT}

This paper is supported by The University Program of Tianjin University of Technology and Education(KJ1701); Graduate Innovation Fund Project of Research Department of Tianjin Vocational and Technical Normal University(YC1909)Tianjin Enterprise Science and Technology Commissioner Project,Grant number(18JCTPJC67500); The National Science Foundation for Young Scientists of China (601301040); The National Key Technology R\&D Program(2015BAK06B04); The key Technologies R\&D program of Tianjin(15ZXZNGX00260, 17YFCZZC0027, 17KPXMSF00190 , 17KPXMMSF00180); The university program of Tianjin University of Technology and Education(KJ1701)

\section{REFERENCES}

[1] Baodang, Zhang Fulian, Xing Qiuling. Reliability and validity of Chinese version of Multidimensional Fatigue Scale in type 2 diabetic patients [J]. Tianjin Nursing, 2016, 24 (5): 377-379. [3] Tanaka M, Mizuno K, Tajima S, et al. Central nervous system fatigue alters autonomic nerve activity.[J]. Life Sciences, 2009, 84(7):235-239.

[2] Konstandi M, Johnson E, Lang M A, et al. NORADRENALINE, DOPAMINE, SEROTONIN: DIFFERENT EFFECTS OF PSYCHOLOGICAL STRESS ON BRAIN BIOGENIC AMINES IN MICE AND RATS [J]. Pharmacological Research, 2000, 41(3):341346.

[3] Tension is. Distinction and Significance of Psychological Fatigue and Physiological Fatigue [C] National Sports Science Congress. 2007.

[4] Guo Siyuan, Ma Jie, Han Chenxia, et al. Research progress of mental fatigue [J]. Advances in modern biomedicine, 2016, 16 (5): 993-997.

[5] Zhong Ming-en, Huang Jiehong, Qiao Yunhao, et al. Comparison of the effects of physical fatigue and mental fatigue on vehicle driving [J]. China Safety Production Science and Technology, 2017, 13 (1): 22-27. 